\title{
Combined effect of hyperhomocysteinemia and smoking on the severity of coronary artery disease in young adults $\leq 35$ years of age: a hospital-based observational study
}

Jiayin Sun, Wei Han, Sijing Wu, Shuo Jia, Zhenxian Yan, Yonghe Guo, Yingxin Zhao, Yujie Zhou and Xiaoli Liu*

\begin{abstract}
Background: The prevalence of coronary artery disease (CAD) continues to increase among young Chinese adults. Current smoking has been recognized as a major risk factor for premature CAD, and hyperhomocysteinaemia (HHcy) has also been suggested to be associated with CAD progression. However, the combined effect of current smoking and HHcy on the severity of coronary artery stenosis in young adults is still uncertain.

Methods: We consecutively collected young patients (18-35 years of age), diagnosed with CAD and underwent coronary angiography (CAG) at Anzhen Hospital between January 2013 and May 2020. HHcy was defined as serum homocysteine (Hcy) level > 15 mol/L. The severity of coronary artery stenosis was evaluated by Gensini Score. The co-effect of current smoking and HHcy on CAD severity as well as the relationship between plasma Hcy, pack-years of smoking and CAD severity were assessed by multivariate linear regression analysis.

Results: A total of 989 participants (mean age, 33 years; $96.2 \%$ male) fulfilling the criteria were enrolled in this study. Patients with both HHcy and current smoking accounted for $39.1 \%$ of all the subjects. Multivariate liner analysis indicated both serum Hcy levels $(\beta 0.302 ; 95 \% \mathrm{Cl} 0.141-0.462 ; P<0.001)$ and pack-years of smoking $(\beta 0.523 ; 95 \% \mathrm{Cl}$ $0.265-0.781 ; P<0.001$ ) were independently associated with the severity of coronary artery stenosis after adjusting for other traditional confounders. In addition, serum Hcy levels were correlated with pack-years of smoking in young CAD patients $(r=0.116, P=0.001)$. Moreover, combination of HHcy and current smoking was suggested to have higher risk for $C A D$ severity $(\beta 17.892 ; 95 \% \mathrm{Cl} 11.314-24.469 ; P<0.001)$, compared with HHcy $(\beta 7.471 ; 95 \% \mathrm{Cl} 0.009-14.934$; $P=0.048)$ or current smoking $(\beta 7.421 ; 95 \% \mathrm{Cl} 0.608-14.233 ; P=0.033)$ alone.
\end{abstract}

Conclusion: Combination of HHcy and smoking is independently associated with the severity of CAD in young patients $\leq 35$ years of age.

Keywords: Coronary artery disease, Hyperhomocysteinemia, Smoking, Severity, Young

*Correspondence: liux19881@163.com

Department of Cardiology, Beijing Anzhen Hospital, Capital Medical University, Beijing Institute of Heart Lung and Blood Vessel Disease, Beijing 100029, China

\section{Introduction}

Coronary artery disease (CAD), with a continuously rising prevalence and mortality, has become a significant public health problem in China [1]. Due to the changes in lifestyle, including the increased obesity rate and reduced physical exercise, the onset age for CAD has gradually gone down, while young CAD patients with onset 
age $\leq 45$ years has gradually increased [2, 3]. Several traditional risk factors, such as age, current smoking status, diabetes mellitus (DM), and increased low-density lipoprotein cholesterol (LDL-C) level, have been identified to be correlated with the severity of CAD among young patients $[4,5]$. Current smoking, considering as the most frequent and one of the most important risk factors for CAD in young adults, exerts a deleterious impact via impairing endothelial function. Moreover, some studies indicated hyperhomocysteinemia (HHcy), as a non-traditional risk factor, was positively associated with severe CAD by increasing lesion counts in patients with premature CAD, which was also due to the vascular endothelial damage and dysfunction [6]. In addition, previous studies have investigated the relationship between plasma Hcy and smoking. Cigarette smoking was showed to increase serum Hcy levels and decrease folate and vitamin B12 levels, which was strongly correlated with the duration of use and the number of cigarettes consumed [7]. Another study also indicated pack-years of smoking were significantly positively correlated with serum Hcy levels, which may due to the effect of oxidative damage [8].

Considering the adverse effects of HHcy and smoking exerting on endothelial function and atherosclerotic disease, along with the obvious correlation between the two factors, investigating the co-effect of HHcy and current smoking on the severity of premature CAD is demanded. Thus, the current study was conducted to determine the following: (1) the combined effect of HHcy and current smoking on the severity of coronary artery stenosis (assessed with a Ginsini scoring system) in young adults $\leq 35$ years of age; (2) the association between CAD severity and both serum Hcy levels and pack-years of smoking in the study population.

\section{Methods}

\section{Study population}

In this observational study, we consecutively collected young patients (18-35 years of age), diagnosed with CAD and underwent coronary angiography (CAG) at Anzhen Hospital between January 2013 and May 2020. The exclusion criteria were as follows: repeated hospitalization, missing homocysteine data, and smoking cessation for more than 1 year. Patients with severe renal insufficiency, hypothyroidism, psoriasis, multiple arteritis, Kawasaki disease, rheumatic heart disease, myocarditis, infective endocarditis, congenital heart disease, cardiomyopathy, valvular heart disease, or having vitamin or folate supplementation within three months were also excluded. This study was approved by the Institutional Ethics Committee of Beijing Anzhen Hospital. The demographic and clinical data we used were retrospectively obtained from electronic medical records.

\section{Definitions and grouping}

Serum levels of Hcy $>15$ umol/L was defined as HHcy [9]. Occasional or regular smoking $\geq 1 \mathrm{cig} /$ day was defined as smoking status, which also included former smokers with cessation period $\leq 1$ year [10]. Pack-years of smoking was defined as the average number of packs of cigarettes smoked per day multiplying the number of years of smoking. Hypertension was defined as a systolic blood pressure $(\mathrm{SBP}) \geq 140 \mathrm{mmHg}$ and/or diastolic blood pressure (DBP) $\geq 90 \mathrm{mmHg}$, or using antihypertensive medications currently [11]. Diabetic mellitus (DM) was defined as fasting blood glucose $(\mathrm{FBG}) \geq 7.0 \mathrm{mmol} / \mathrm{L}$ and/or random glucose level $\geq 11.1 \mathrm{mmol} / \mathrm{L}$, or previously diagnosed DM with the treatment of diet, oral agents, or insulin [12]. Hypertriglyceridemia was defined as triglycerides $(\mathrm{TG}) \geq 1.7 \mathrm{mmol} / \mathrm{L}$, hypercholesterolemia was defined as total cholesterol (TC) $\geq 5.2 \mathrm{mmol} / \mathrm{L}$, a high low-density lipoprotein cholesterol (LDL-C) level was defined as $\mathrm{LDL}-\mathrm{C} \geq 3.4 \mathrm{mmol} / \mathrm{L}$, and a low high-density lipoprotein cholesterol (HDL-C) level was defined as $\mathrm{HDL}-\mathrm{C}<1.0 \mathrm{mmol} / \mathrm{L}$ [13]. Serum uric acid (UA) level $\geq 420 \mathrm{mmol} / \mathrm{L}$ in males and $\geq 357 \mathrm{mmol} / \mathrm{L}$ in females was considered to be hyperuricemia [14]. Someone with average alcohol intake $\geq 50 \mathrm{~g} /$ day was considered to be drinker.

The study population was divided into four groups according to presence or absence of HHcy and smoking status, which were as follows: HHcy - Smoker - group, HHcy+Smoker - group, HHcy-Smoker + group and HHcy+Smoker+ group.

All patients received coronary angiography using a standard technique. A luminal diameter stenosis $\geq 50 \%$ in any of the major coronary arteries, including the left main, left circumflex, left anterior descending, right coronary artery, and main branches with a diameter of more than $2.0 \mathrm{~mm}$, was defined as CAD. In addition, patients who diagnosed as acute myocardial infraction were also considered to have CAD. Left main stenosis $\geq 50 \%$ was considered as a double-vessel disease. Multivessel disease was defined as $\geq 50 \%$ stenosis in more than one major coronary vessels. Gensini Score was used to quantify the severity of CAD [15]. Based on the results of CAG, the score of each lesion was calculated by severity score, which reflected the stenosis degree of luminal narrowing and modified by the collateral adjustment factor, multiplying region factor, which reflected the geographic importance of the lesion location in the coronary tree. The final Gensini Score was expressed as the sum of all the lesion scores.

\section{Data collection}

Baseline venous blood samples were taken from all participants after an overnight fast within the first $24 \mathrm{~h}$ of 
hospitalization. The following biochemical parameters were analyzed: TG, TC, LDL-C, HDL-C, UA, blood urea nitrogen (BUN), creatinine (CR), glycated hemoglobin (HbA1c) and high-sensitivity $\mathrm{C}$ - reactive protein (hs-CRP). Hcy commercial kit (enzymatic cycling method) was used to test serum Hcy levels by a Beckman Coulter AU5400 automatic biochemical analyzer.

The clinical data of participants, including age, gender, body mass index (BMI), history of hypertension and DM, family history of CAD as well as smoking and drinking status, were retrospectively obtained from electronic medical records.

\section{Statistical analysis}

All the analysis was conducted by the statistical software SPSS 22.0 (IBM-SPSS Inc., Chicago, USA). Kolmogorov-Smirnov test was used to evaluate the normality of continuous data. Accordingly, normally distributed continuous variables were presented as mean \pm standard deviation, and non-normally distributed data as median [interquartile (25th-75th percentiles) range]. Where indicated, one-way analysis of variance and Kruskal-Wallis $\mathrm{H}$ test were applied to evaluate statistical differences among groups with different Hcy levels and smoking status. Then, pairwise comparison was performed among groups using $\mathrm{S}-\mathrm{N}-\mathrm{K}$ test or Mann-Whitney U test. Categorical variables were expressed as counts and percentages (\%), and differences among these groups were examined by Chi-square test. The relationship between serum Hcy levels and pack-years of smoking was evaluated using Spearman analysis. Variables with a $P$ value $<0.1$ in the Univariate liner regression analysis as well as the traditional risk factors for CAD were selected and added into multivariate liner regression analysis to determine their effects on the severity of CAD, which was calculated by Beta with $95 \%$ confidence intervals $(95 \% \mathrm{CI})$. Moreover, the relationship of Hcy, pack-years of smoking and the severity of CAD was also assessed by multivariate linear regression analysis. $P$ values of $<0.05$ in a two-sided test was considered statistically significant.

\section{Results}

Baseline clinical characteristics

As shown in Fig. 1, a total of 989 participants fulfilling the criteria were enrolled in this study (mean age,

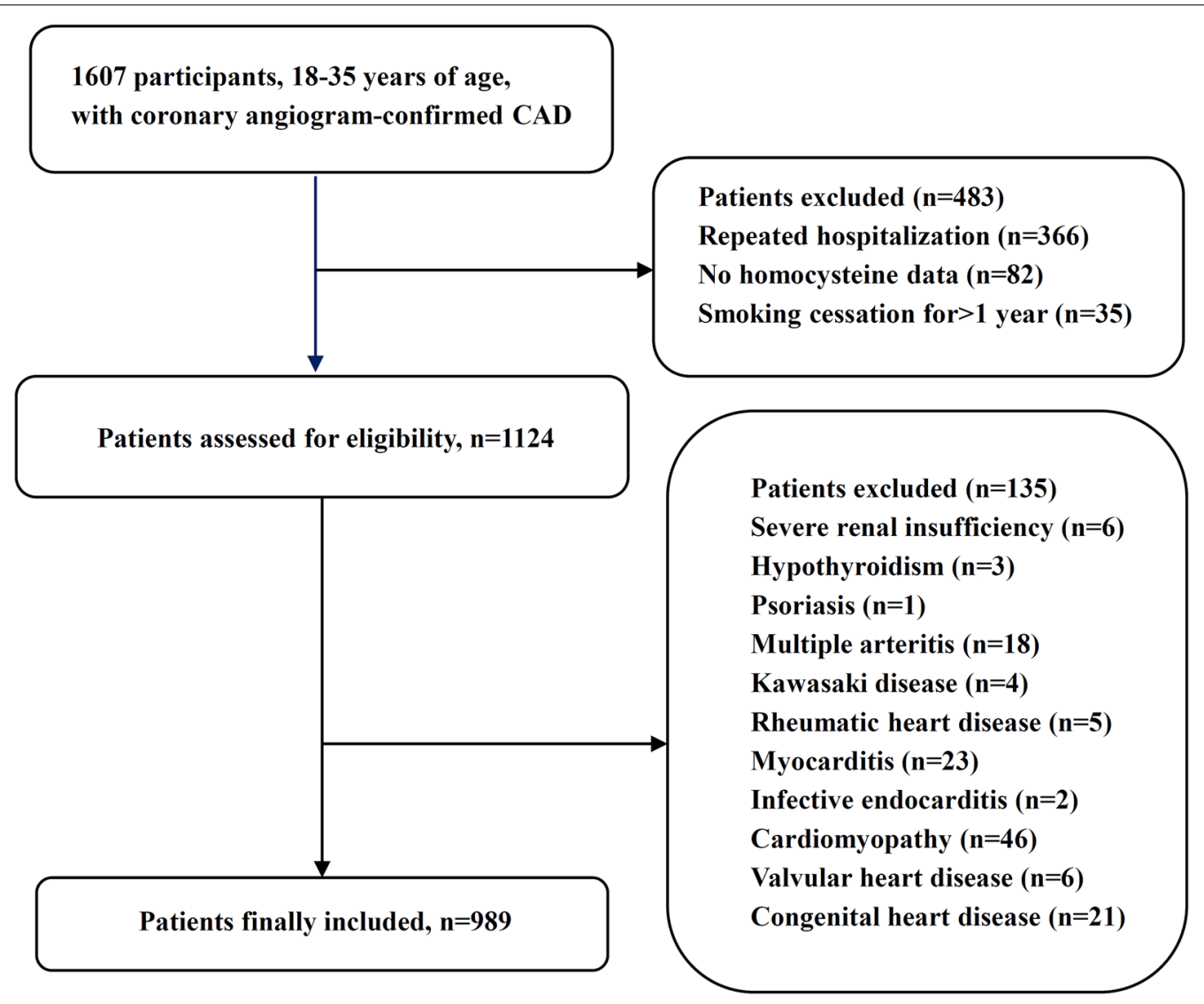

Fig. 1 Flow chart illustrating the process of a participant enrolled in the study 
Table 1 Baseline clinical characteristics of study population

\begin{tabular}{|c|c|c|c|c|c|}
\hline Characteristics & Total $(n=989)$ & $\begin{array}{l}\text { HHcy-Smoker- } \\
(n=153)\end{array}$ & $\begin{array}{l}\text { HHcy+Smoker- } \\
(n=160)\end{array}$ & $\begin{array}{l}\text { HHcy- } \\
\text { Smoker+(n=289) }\end{array}$ & HHcy + Smoker $+(n=387)$ \\
\hline Age (years) & $33(30-34)$ & $33(30-34)$ & $32(29-34)$ & $33(31-34)$ & $32(30-34)$ \\
\hline Male, n (\%) & 951 (96.16) & $128(83.66)$ & $151(94.38)^{\mathrm{a}}$ & $288(99.65)^{\mathrm{ab}}$ & $384(99.22)^{a b}$ \\
\hline $\mathrm{SBP}(\mathrm{mmHg})$ & $126.44 \pm 15.61$ & $126.87 \pm 14.97$ & $127.44 \pm 14.94$ & $127.36 \pm 16.71$ & $125.18 \pm 15.25$ \\
\hline $\mathrm{DBP}(\mathrm{mmHg})$ & $78.01 \pm 13.19$ & $78.21 \pm 14.18$ & $78.43 \pm 14.13$ & $78.25 \pm 13.71$ & $77.57 \pm 11.97$ \\
\hline Heart rate (bpm) & $75.94 \pm 11.71$ & $75.34 \pm 10.17$ & $77.25 \pm 11.36$ & $76.21 \pm 12.14$ & $75.43 \pm 12.07$ \\
\hline Drinker, n (\%) & $182(18.40)$ & $14(9.15)$ & $9(5.63)$ & $80(27.68)^{\mathrm{ab}}$ & $79(20.41)^{\mathrm{ab}}$ \\
\hline \multicolumn{6}{|l|}{$\begin{array}{l}\text { Medical history and } \\
\text { coronary risk factors }\end{array}$} \\
\hline BMI $\left(\mathrm{kg} / \mathrm{m}^{2}\right)$ & $28.34 \pm 5.01$ & $27.68 \pm 6.15$ & $28.09 \pm 4.57$ & $28.72 \pm 5.08$ & $28.44 \pm 4.57$ \\
\hline Hypertension, n (\%) & 485 (49.04) & $74(48.37)$ & $77(48.13)$ & $149(51.56)$ & $185(47.80)$ \\
\hline Diabetes mellitus, n (\%) & $191(19.31)$ & $36(23.53)$ & $25(15.63)$ & $82(28.37)^{b}$ & $48(12.40)^{\mathrm{ac}}$ \\
\hline $\begin{array}{l}\text { Hypertriglyceridemia, } \\
\text { n (\%) }\end{array}$ & $559(56.52)$ & $65(42.48)$ & $82(51.25)$ & $178(61.59)^{\mathrm{a}}$ & $234(60.47)^{\mathrm{a}}$ \\
\hline $\begin{array}{l}\text { Hypercholesterolemia, } \\
\text { n (\%) }\end{array}$ & $336(33.97)$ & $51(33.33)$ & $45(28.13)$ & $114(39.45)$ & $126(32.56)$ \\
\hline High LDL-C, n (\%) & $307(31.04)$ & $37(24.18)$ & $42(26.25)$ & $104(35.99)$ & $124(32.04)$ \\
\hline Low HDL-C, n (\%) & $677(68.45)$ & $96(62.75)$ & $106(66.25)$ & $200(69.20)$ & $275(71.06)$ \\
\hline $\begin{array}{l}\text { Family history of CAD, } \\
\mathrm{n}(\%)\end{array}$ & $127(12.84)$ & $13(8.50)$ & $8(5.00)$ & $42(14.53)^{b}$ & $64(16.54)^{b}$ \\
\hline Hyperuricemia, n (\%) & 437 (44.19) & $61(39.87)$ & $71(44.38)$ & 119 (41.18) & $186(48.06)$ \\
\hline Prior stroke, n (\%) & $5(0.51)$ & $0(0.00)$ & $0(0.00)$ & $3(1.00)$ & $2(0.50)$ \\
\hline \multicolumn{6}{|l|}{ Laboratory results } \\
\hline BUN (mmol/L) & $4.73 \pm 1.31$ & $4.67 \pm 1.46$ & $4.77 \pm 1.31$ & $4.66 \pm 1.17$ & $4.79 \pm 1.31$ \\
\hline$C R(\mu \mathrm{mol} / \mathrm{L})$ & $74.78 \pm 15.12$ & $72.21 \pm 14.81$ & $77.64 \pm 19.96^{\mathrm{a}}$ & $72.58 \pm 12.39^{b}$ & $76.24 \pm 14.39^{\mathrm{ac}}$ \\
\hline HbA1c (\%) & $5.6(5.3-6.2)$ & $5.6(5.2-6.7)$ & $5.5(5.2-6.1)$ & $5.7(5.4-6.8)^{b}$ & $5.5(5.2-5.9)^{c}$ \\
\hline $\mathrm{TG}(\mathrm{mmol} / \mathrm{L})$ & $1.88(1.30-2.85)$ & $1.47(1.01-2.25)$ & $1.75(1.15-2.32)$ & $2.05(1.39-3.36)^{\mathrm{ab}}$ & $2.02(1.43-2.93)^{\mathrm{ab}}$ \\
\hline TC (mmol/L) & $4.74 \pm 1.73$ & $4.64 \pm 2.10$ & $4.66 \pm 2.01$ & $4.92 \pm 1.67$ & $4.69 \pm 1.46$ \\
\hline $\mathrm{HDL}-\mathrm{C}(\mathrm{mmol} / \mathrm{L})$ & $0.92 \pm 0.25$ & $0.95 \pm 0.23$ & $0.93 \pm 0.21$ & $0.92 \pm 0.35$ & $0.90 \pm 0.19$ \\
\hline LDL-C (mmol/L) & $3.03 \pm 1.56$ & $2.95 \pm 1.97$ & $3.01 \pm 1.91$ & $3.13 \pm 1.49$ & $2.99 \pm 1.23$ \\
\hline UA $(\mu \mathrm{mol} / \mathrm{L})$ & $411.23 \pm 97.88$ & $390.73 \pm 101.55$ & $417.69 \pm 107.07^{\mathrm{a}}$ & $405.44 \pm 83.03$ & $420.99 \pm 101.54^{\mathrm{a}}$ \\
\hline $\operatorname{Hcy}(\mu \mathrm{mol} / \mathrm{L})$ & $15.60(11.30-26.60)$ & $10.30(8.70-12.20)$ & $22.95(16.53-33.38)^{a}$ & $11.00(9.00-13.10)^{\mathrm{ab}}$ & $25.80(17.80-39.40)^{\mathrm{abc}}$ \\
\hline hs-CRP (mg/L) & $2.70(0.97-8.93)$ & $1.37(0.54-4.85)$ & $2.55(0.97-6.10)^{\mathrm{a}}$ & $3.11(1.27-11.29)^{\mathrm{ab}}$ & $3.12(0.95-12.91)^{\mathrm{ab}}$ \\
\hline
\end{tabular}

Data are expressed as mean \pm standard deviation, medians with interquartile range or number (\%)

$C A D$ coronary artery disease, $H H c y$ hyperhomocysteinemia, $S B P$ systolic blood pressure, $D B P$ diastolic blood pressure, $B M I$ body mass index, $B U N$ blood urea nitrogen, $C R$ creatinine, $H b A 1 c$ glycated hemoglobin, $T G$ triglyceride, $T C$ total cholesterol, $H D L-C$ high-density lipoprotein cholesterol, $L D L-C$ low-density lipoprotein cholesterol, $U A$ uric acid, $h s-C R P$ high-sensitivity $C$-reactive protein

${ }^{a} p<0.05$ versus $\mathrm{HHcy}-$ Smoker-

${ }^{\mathrm{b}} p<0.05$ versus $\mathrm{HHcy}+$ Smoker-

${ }^{c} p<0.05$ versus $\mathrm{HHcy}-$ Smoker+

33 years; $96.2 \%$ male). The baseline characteristics and biochemical findings of all the patients in four groups are listed in Table 1. Patients with HHcy accounted for $55.3 \%$ and patients with current smoking accounted for $68.4 \%$, while patients with both HHcy and current smoking accounted for $39.1 \%$ of all the young participants. Smokers (both in HHcy-Smoker+group and HHcy+Smoker+group) had a higher prevalence of male gender, drinker, hypertriglyceridemia and family history of CAD, as well as increased levels of TG and hs-CRP compared with non-smokers (both in HHcy-Smokergroup and HHcy+Smoker- group). Meanwhile, the HHcy+Smoker- group also had higher male prevalence and elevated hs-CRP levels compared with the HHcySmoker- group, but not as significant as smokers. Moreover, patients with HHcy (both in HHcy+Smokergroup and HHcy+Smoker+group) had increased levels of CR and UA, but a lower prevalence of DM compared with patients without HHcy (both in HHcy-Smokergroup and HHcy-Smoker+group). 
Table 2 Clinical diagnosis, angiographic findings, medical treatment and cardiac function of study population

\begin{tabular}{|c|c|c|c|c|c|}
\hline Characteristics & Total $(\mathrm{n}=989)$ & $\begin{array}{l}\text { HHcy-Smoker- } \\
(n=153)\end{array}$ & $\begin{array}{l}\text { HHcy+Smoker- } \\
(n=160)\end{array}$ & $\begin{array}{l}\text { HHcy-Smoker }+ \\
(n=289)\end{array}$ & $\begin{array}{l}\text { HHcy+Smoker }+ \\
(n=387)\end{array}$ \\
\hline \multicolumn{6}{|l|}{ Diagnosis } \\
\hline SCAD & $102(10.31)$ & $32(20.92)$ & $13(8.13)^{\mathrm{a}}$ & $33(11.42)^{\mathrm{a}}$ & $24(6.20)^{\mathrm{a}}$ \\
\hline UAP & $379(38.32)$ & $74(48.37)$ & $67(41.88)$ & $97(33.56)^{\mathrm{a}}$ & $141(36.43)$ \\
\hline AMI & $508(51.37)$ & $47(30.72)$ & $80(50.00)^{\mathrm{a}}$ & $159(55.02)^{\mathrm{a}}$ & $222(57.36)^{a}$ \\
\hline \multicolumn{6}{|c|}{ Angiographic findings of vessel involvement } \\
\hline None & $48(4.85)$ & $17(11.11)$ & $11(6.88)$ & $16(5.54)$ & $4(1.03)^{a b c}$ \\
\hline Single vessel & $447(45.20)$ & $66(43.14)$ & $70(43.75)$ & $134(46.37)$ & $177(45.74)$ \\
\hline Double vessel & $236(23.86)$ & $40(26.14)$ & $37(23.13)$ & $60(20.76)$ & $99(25.58)$ \\
\hline Triple vessel & $258(26.09)$ & $30(19.61)$ & $42(26.25)$ & $79(27.33)$ & $107(27.65)$ \\
\hline Multi-vessel & $494(49.95)$ & $70(45.75)$ & $79(49.38)$ & $139(48.1)$ & $206(53.23)$ \\
\hline \multicolumn{6}{|l|}{ Treatment } \\
\hline Drug & $211(21.33)$ & $51(33.33)$ & $33(20.63)^{a}$ & $54(18.69)^{\mathrm{a}}$ & $73(18.86)^{\mathrm{a}}$ \\
\hline Intervention & $754(76.24)$ & $100(65.36)$ & $124(77.50)^{\mathrm{a}}$ & $228(78.89)^{\mathrm{a}}$ & $302(78.04)^{\mathrm{a}}$ \\
\hline Coronary artery bypass grafting & $24(2.43)$ & $2(1.31)$ & $3(1.87)$ & $7(2.42)$ & $12(3.10)$ \\
\hline \multicolumn{6}{|l|}{ Cardiac function } \\
\hline LVEF & $60(55-66)$ & $62(58-67)$ & $61(53-66)$ & $61(55-66)$ & $60(53-65)^{\mathrm{ac}}$ \\
\hline Gensini Score & $34(20-62)$ & $24(11-43)$ & $32(13-57.5)^{\mathrm{a}}$ & $32(18.5-50)^{\mathrm{a}}$ & $44(24-78)^{\mathrm{abc}}$ \\
\hline
\end{tabular}

Data are expressed as the number (\%)

$S C A D$ stable coronary artery disease, UAP unstable angina pectoris, $A M I$ acute myocardial infraction, HHcy hyperhomocysteinemia, $L V E F$ left ventricular ejection fraction

${ }^{\mathrm{a}} p<0.05$ versus $\mathrm{HHcy}-$ Smoker-

${ }^{\mathrm{b}} p<0.05$ versus $\mathrm{HH}$ cy + Smoker-

${ }^{c} p<0.05$ versus $\mathrm{HHcy}-$ Smoker +

Table 3 Univariate liner regression analysis of the severity of CAD with variables

\begin{tabular}{lccc}
\hline Variables & $\boldsymbol{\beta}$ & $\mathbf{9 5 \%} \mathbf{C l}$ & $\boldsymbol{P}$ \\
\hline Age & -0.406 & $-1.097,0.284$ & 0.249 \\
Male & 6.102 & -4.304 to 16.509 & 0.250 \\
BMl & 0.002 & -0.436 to 0.440 & 0.993 \\
Drinker & -6.805 & -11.952 to -1.657 & $\mathbf{0 . 0 1}$ \\
Smoker & 9.575 & $5.321-13.829$ & $<\mathbf{0 . 0 0 1}$ \\
Hypertension & 0.878 & -3.126 to 4.881 & 0.667 \\
Diabetes mellitus & 3.696 & -1.369 to 8.762 & 0.152 \\
Hypertriglyceridemia & 4.686 & $0.659-8.713$ & $\mathbf{0 . 0 2 3}$ \\
Hypercholesterolemia & 4.127 & -0.091 to 8.346 & 0.055 \\
High LDL-C & 8.185 & $3.890-12.481$ & $<\mathbf{0 . 0 0 1}$ \\
Low HDL-C & 4.304 & $0.005-8.603$ & $\mathbf{0 . 0 4 9}$ \\
Family history of CAD & 0.294 & -5.698 to 1.973 & 0.206 \\
Hyperhomocysteinemia & 11.463 & $7.496-15.429$ & $<\mathbf{0 . 0 0 1}$ \\
Uric acid & 0.026 & $0.006-0.047$ & $\mathbf{0 . 0 1 3}$ \\
BUN & 0.025 & -0.056 to 0.107 & 0.543 \\
CR & 0.057 & -0.012 to 0.125 & 0.105 \\
\hline
\end{tabular}

Bold values indicate statistical significance

$C A D$ coronary artery disease, $B M I$ body mass index, $H D L-C$ high-density lipoprotein cholesterol, $L D L-C$ low-density lipoprotein cholesterol, $B U N$ blood urea nitrogen, $C R$ creatinine, $\mathrm{Cl}$ confidence interval
In addition, stable coronary artery disease (SCAD) diagnosis and drug treatment were more prevalent in patients of HHcy-Smoker- group, while AMI diagnosis and coronary intervention were more prevalent in the counterparts of the other three groups. Analysis of the CAG findings demonstrated that there was no difference on the numbers of lesion vessels among four groups, except for having a smaller proportion of patients with no lesion vessels in the HHcy+Smoker+group. Besides, the highest value of Gensini Score was observed in the HHcy+Smoker+group, while the lowest was found in the HHcy-Smoker- group [44 (24-78) in HHcy+Smoker+group vs. 32 (18.5-50) in HHcySmoker+group or 32 (13-57.5) in HHcy+Smokergroup vs. 24 (11-43) in HHcy-Smoker - group, $P<0.05$ respectively]. Patients in the HHcy+Smoker+group also had a relatively lower LVEF level (Table 2).

\section{Univariate analysis of different CAD risk factors}

As shown in Table 3, traditional CAD risk factors such as current smoker status, hypertriglyceridemia, High LDL-C and low HDL-C were apparently associated with the severity of CAD $(P<0.05)$, while drinker showed a negative correlation. On the contrary, univariate analysis also showed that age, male gender, BMI, hypertension, DM, 


\begin{tabular}{|c|c|c|c|c|c|}
\hline Variable & & & B & $(95 \% \mathrm{Cl})$ & P value \\
\hline Age & & 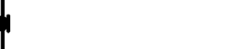 & -0.147 & $(-0.921,0.627)$ & 0.709 \\
\hline BMI & & & -0.163 & $(-0.595,0.269)$ & 0.458 \\
\hline Drinker & $\longmapsto$ & & -9.493 & $(-15.134,-3.853)$ & 0.001 \\
\hline Hypertension & 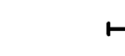 & $\longrightarrow$ & 0.434 & $(-3.988,4.856)$ & 0.847 \\
\hline Diabetes mellitus & & $\longmapsto$ & 8.31 & $(2.656,13.965)$ & 0.004 \\
\hline Hypertriglyceridemia & & fo- & 1.916 & $(-2.721,6.552)$ & 0.418 \\
\hline Hypercholesterolemia & & $\longrightarrow$ & -2.637 & $(-9.720,4.447)$ & 0.465 \\
\hline High LDL-C & & $\longmapsto$ & 9.022 & $(1.876,16.168)$ & 0.013 \\
\hline Low HDL-C & & $\longrightarrow$ & 3.998 & $(-0.765,8.761)$ & 0.1 \\
\hline Uric acid & & & 0.01 & $(-0.013,0.033)$ & 0.39 \\
\hline Family history of CAD & & $\longrightarrow$ & -2.054 & $(-8.545,4.437)$ & 0.535 \\
\hline HHcy+Smoker+ & & $\longmapsto$ & 17.892 & $(11.314,24.469)$ & $<0.001$ \\
\hline HHcy-Smoker+ & & $\longrightarrow$ & 7.421 & $(0.608,14.233)$ & 0.033 \\
\hline HHcy+Smoker- & & $\longrightarrow$ & 7.471 & $(0.009,14.934)$ & 0.048 \\
\hline HHcy-Smoker- & & & (Referemce) & (Referemce) & \\
\hline & -10 & 20 & & & \\
\hline
\end{tabular}

Fig. 2 Forest plot of multivariate linear regression analysis of the severity of CAD with different CAD risk factors. CAD coronary artery disease, BMI body mass index, HDL-C high-density lipoprotein cholesterol, LDL-C low-density lipoprotein cholesterol, HHcy hyperhomocysteine, Cl confidence interval

hypercholesterolemia and family history of CAD had no significant association with the severity of CAD. Moreover, non-traditional risk factors, such as UA, especially HHcy, were also obviously related to the severity of CAD (for HHcy, $\beta$ 11.463; 95\% CI 7.496-15.429; $P<0.001$ ).

\section{Multivariate liner regression analysis of different CAD risk factors}

Except for the variables with $P<0.1$ in the univariate analysis, traditional CAD risk factors with $P>0.1$, including age, BMI, hypertension, DM and family history of CAD were also added in the Multivariate liner regression analysis. The results indicated that $\mathrm{DM}(\beta$ 8.310; 95\% CI 2.65613.965; $P=0.004)$ and high LDL-C $(\beta$ 9.022; $95 \% \mathrm{CI}$ $1.876-16.168 ; P=0.013)$ were the significant risk factors, while drinker was a protective factor for the severity of CAD among young adults, after adjusting for confounding factors. Moreover, patients with both HHcy and smoking had 17.892-fold risk for CAD severity (95\% CI 11.31424.469) compared with those without HHcy and smoking, and had more than two times risk for the severity of CAD, as compared to patients with either HHcy or smoking (for HHcy-Smoker+, $\beta$ 7.421; 95\% CI 0.608-14.233; for HHcy+ Smoker-, $\beta$ 7.471; 95\% CI 0.009-14.934) (Fig. 2).

\section{Correlation of serum Hcy levels with Pack-years of smoking in young CAD patients}

Figure 3 showed that serum Hcy levels were apparently correlated with pack-years of smoking in young CAD patients $(r=0.116, P=0.001)$.
Multivariate analysis of CAD severity with serum Hcy levels and pack-years of smoking

Multivariate liner regression analysis was performed to determine the correlation of CAD severity and serum Hcy levels as well as pack-years of smoking. As shown in Table 4, after adjusting for BMI, drinker, hypertension, DM, triglyceride, HDL-C and LDL-C, both serum Hcy levels $(\beta$ 0.302; 95\% CI 0.141-0.462; $P<0.001)$ and packyears of smoking $(\beta 0.523$; 95\% CI $0.265-0.781 ; P<0.001)$ were significantly associated with the severity of CAD in young adults.

\section{Discussion}

To our knowledge, this observational study firstly investigated the co-effect of HHcy and current smoking on the CAD severity in patients less than 35 years of age. From this large-scale study, we found that the combination of HHcy and smoking, acting as the most important risk factor, was independently associated with the severity of coronary artery stenosis among young subjects.

Young patients with CAD represent a minority proportion, about $6-10 \%$ adults below 40 years of age are diagnosed with $\mathrm{CAD}$, but the incidence has been obviously increased [16]. Among the variety of conventional risk factors involved in the progression of premature CAD, current smoking was considered to be the most frequent one, which exerted significant deleterious effect [17]. The amount of cigarette smoking, especially the pack-years of smoking, have been shown to be associated with vascular disease. A study involving 416 middle-aged and 


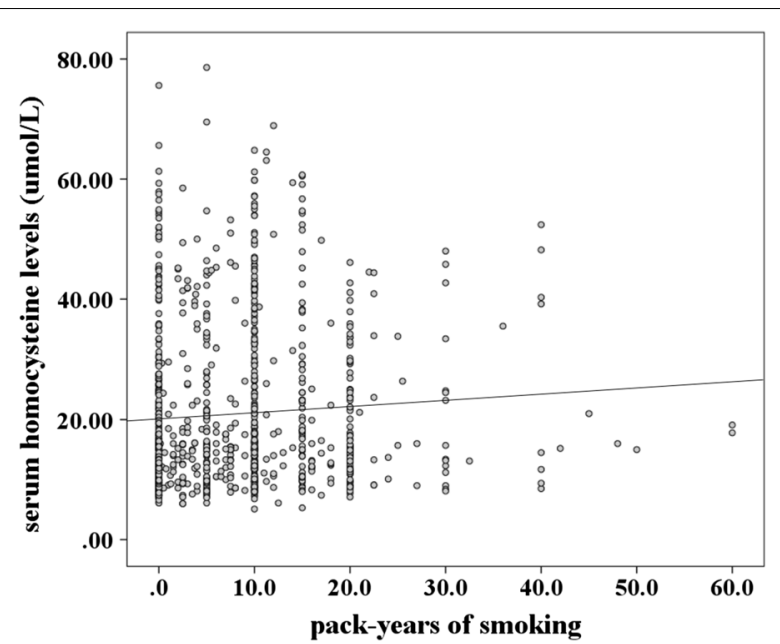

Fig. 3 Correlation of serum homocysteine levels with pack-years of smoking $(r=0.116, P=0.001)$

elderly patients showed Pack-years might be the most important factor that was correlated with the severity of coronary stenosis in terms of Gensini Score $(r=0.193$, $P=0.013)[18]$. As for younger subjects, Cheezum et al. [19] enrolled a population with a median age 50 years and suggested a dose-dependent (Pack-years $\geq 12$ ) association between smoking and extent of plaque. In the current study, we firstly indicated a positive correlation between pack-years of smoking and severity of coronary atherosclerosis in young patients $\leq 35$ years of age, which was independent of the other conventional risk factors $(\beta$ 0.523; 95\% CI 0.265-0.781; $P<0.001)$. Furthermore, the risk due to smoking was shown to last up to 20 years for CAD after smoking cessation, but since 1 year cessation was significantly associated with $2 \%$ lower risk for CAD on average [20], young patients with smoking cessation for more than 1 year were excluded in the study.

Hcy level is a well-known biomarker of CAD and elevated concentrations of Hcy have been identified to be a crucial promoter for atherosclerotic vascular disease. However, some controversy exists regarding the causal role of Hcy in CAD pathogenesis, since lowering Hcy levels in patients with CAD has not shown any benefit and common genetic variants that influence Hcy levels are not associated with risk of CAD [21, 22]. Nevertheless, several observational studies found HHcy, as an important marker, was significantly associated with severe CAD in younger patients $[6,23]$. In this study, the relationship between concentrations of Hcy and the severity of coronary artery stenosis was also investigated, which showed that serum Hcy levels were positively associated with angiographic severity expressed by Gensini Score $(\beta$ 0.302 ; $95 \%$ CI $0.141-0.462 ; P<0.001)$. Our data were consistent with the results of previous study conducted by Karadeniz and colleagues [24], which enrolled 503 elderly patients with acute coronary syndrome, and suggested Hcy levels were independently related to the severity of CAD ( $\beta$ 0.144; $P=0.017)$. However, the Karadeniz et al. [24] study showed no significant effect of smoking on atherosclerotic plaque progression, which was not the same as ours, considering the differences in age and health status of the study participants.

In our study, after eliminating the impact of other CAD risk factors, both serum Hcy levels as well as pack-years of smoking were independently associated with Gensini Score, which indicated combined consideration of Hcy levels and amount of smoking could better reflect the development of CAD. In addition, the results also suggested patients with both HHcy and current smoking had higher risk for CAD severity, compared with those without or with just one factor (HHcy or current smoking). Previous study analyzed the influences of smoking and Hcy levels on the degree of arteriolar retinopathy, which was associated with greater cardiovascular risk, and the results showed in subjects with grade II retinopathy, smoking was related to increased plasma Hcy levels and could independently contribute to the progression of arteriolar retinopathy [25], reflecting the positive effect of smoking and Hcy levels on microvascular disease. Moreover, we found young CAD patients with both HHcy and smoking had decreased value of LVEF, which might be due to the relatively increased Gensini Score and more myocardial ischemia.

Several possible mechanisms might explain the combined effect of HHcy and current smoking on the

Table 4 Multivariate analysis of the severity of CAD with homocysteine and pack-years of smoking

\begin{tabular}{|c|c|c|c|c|c|c|}
\hline \multirow[b]{2}{*}{ Variables } & \multicolumn{3}{|c|}{ Model $1^{\mathrm{a}}$} & \multicolumn{3}{|c|}{ Model $1^{b}$} \\
\hline & $\beta$ & $95 \% \mathrm{Cl}$ & $P$ & $\bar{\beta}$ & $95 \% \mathrm{Cl}$ & $P$ \\
\hline Pack-years of smoking & 0.398 & $0.167,0.428$ & 0.001 & 0.523 & $0.265,0.781$ & $<0.001$ \\
\hline Homocysteine & 0.316 & $0.170,0.462$ & $<0.001$ & 0.302 & $0.141,0.462$ & $<0.001$ \\
\hline
\end{tabular}

Bold values indicate statistical significance

${ }^{\text {a }}$ No other risk factors were adjusted

${ }^{\mathrm{b}}$ Adjusted for body mass index (BMI), drinker, Hypertension, Diabetes mellitus, triglyceride, high-density lipoprotein cholesterol (HDL-C), and low-density lipoprotein cholesterol (LDL-C) 
progression of $\mathrm{CAD}$, since both of these risk factors could exert similar effects. (1) Nicotine and carbon monoxide in tobacco can produce direct vascular endothelial damage by reducing the production of nitric oxide (NO) and prostacyclin, and aggravating the inflammatory reaction [26]. Hcy also have a variety of toxic effects on the vasculature, including impairing endothelial function by decreasing the concentration of NO, inducing vascular remodeling by increasing the smooth muscle cells synthesis, and elevating adventitial inflammation [27]. (2) Both smoking and Hcy may exert a prothrombotic effect via increasing platelet aggregation and destroying the balance of coagulation and fibrinolysis system $[26,28]$. (3) Smoking may promote dyslipidemia, while HHcy may cause Hcy-related LDL atherogenesis, such as small LDL particle size and its oxidative modification $[26,29]$. Those all suggest a strong potential for interaction between two factors in the development of atherosclerosis. In addition, except for damaging the vascular tree independently, smoking and Hcy are also related. Studies have showed that smoking could exert a deleterious impact on plasma Hcy. The pack-years of smoking directly increased the serum Hcy levels through regulating Hcy metabolism by reducing serum folate and vitamin B12 concentrations, as well as activating the systemic oxidation reaction system and damaging the anti-oxidation defense system [7, 8], which indicated the potential effect of smoking on the progression of CAD among young subjects.

The clinical importance of this study is emphasizing the co-effect of current smoking and HHcy on the development and extent of premature $\mathrm{CAD}$, which shows that the combination of these two risk factors has more effect on the severity of CAD among patients $\leq 35$ years of age. From the perspective of CAD prevention, except for stressing the importance of smoking cessation, a young population should pay more attention to the adverse effect of HHcy, and have a healthy lifestyle, such as keeping weight, sufficient vitamin B and folic acid intake, to maintain Hcy levels within the normal range. In addition, serum Hcy levels are associated with smoking in a dose-dependent way, which suggests the potential benefits of smoking cessation in the prevention of CAD among young adults.

\section{Limitations}

Our study has a few limitations. First, although plasma Hcy is mainly determined by vitamin $B$ and folic acid intake, which was also affected by smoking, the levels of vitamin B and folic acid were not measured in this retrospective study. Second, since the majority of young CAD patients were male, the results had limited value for the young female population. Third, Gensini Score, rather than the Syntax Score, was used to evaluate the severity of CAD in the study, since the severity was assessed by CAG reports due to the long interval for participants enrollment. However, Gensini Score was identified to be none inferior to Syntax Score considering the relevance and equivalence [30].

\section{Conclusion}

Combination of HHcy and smoking is independently associated with the severity of CAD in young patients $\leq 35$ years of age.

\begin{abstract}
Abbreviations
CAD: Coronary artery disease; HHcy: Hyperhomocysteinemia; CAG: Coronary angiography; Hcy: Homocysteine; DM: Diabetes mellitus; LDL-C: Low-density lipoprotein cholesterol; SBP: Systolic blood pressure; DBP: Diastolic blood pressure; FBG: Fasting blood glucose; TG: Triglycerides; TC: Total cholesterol; HDL-C: High-density lipoprotein cholesterol; UA: Uric acid; BUN: Blood urea nitrogen; CR: Creatinine; HbA1c: Glycated hemoglobin; hs-CRP: High-sensitivity C-reactive protein; BMI: Body mass index; AMI: Acute myocardial infraction; UAP: Unstable angina pectoris; SCAD: Stable coronary artery disease; LVEF: Left ventricular ejection fraction; Cl: Confidence intervals.
\end{abstract}

\section{Acknowledgements}

We would like to thank our colleagues from the Department of Cardiology, Beijing Anzhen Hospital, Capital Medical University.

\section{Authors' contributions}

Study conception and design: XL, JS. Literature search: JS. Data collection and analysis: JS, WH, SW, SJ, ZY, YG. Data interpretation: XL, YZ, YZ, JS. Writing: JS. All authors read and approved the final manuscript.

\section{Funding}

This work was supported by the National Key Research and Development Program of China (2017YFC0908800), Beijing Municipal Health Commission "Project of Science and Technology Innovation Center" (PXM2019_026272_000006) (PXM2019_026272_000005), Capital's Funds for Health Improvement and Research (CFH2020-2-2063), and Science and technology plan of Beijing Municipal Commission of Education (KM201910025012).

\section{Availability of data and materials}

The datasets used and/or analyzed during the current study are available from the corresponding author on reasonable request.

\section{Declarations}

\section{Ethics approval and consent to participate}

This study was complied with the Declaration of Helsinki and approved by the Institutional Ethics Committee of Beijing Anzhen Hospital. Written informed consent was waived by the ethics committee because of the data retrospectively obtained from electronic medical records.

\section{Consent for publication}

Not applicable.

\section{Competing interests}

The authors declare no competing interests.

Received: 28 February 2021 Accepted: 27 September 2021

Published online: 09 October 2021

\footnotetext{
References

1. Ma LY, Chen WW, Gao RL, Liu LS, Zhu ML, Wang YJ, et al. China cardiovascular diseases report 2018: an updated summary. J Geriatr Cardiol. 2020;17(1):1-8. https://doi.org/10.11909/j.issn.1671-5411.2020.01.001.
} 
2. Blumenthal D, Hsiao W, Blumenthal DM. Caring for coronary artery disease in china: managing modernization. JAMA Intern Med. 2016;176(4):521-3. https://doi.org/10.1001/jamainternmed.2016.0198.

3. Wang X, Gao M, Zhou S, Wang J, Liu F, Tian F, et al. Trend in young coronary artery disease in China from 2010 to 2014: a retrospective study of young patients $\leq 45$. BMC Cardiovasc Disord. 2017;17:18. https://doi.org/ 10.1186/s12872-016-0458-1.

4. Cai G, Liu W, Lv S, Wang X, Guo Y, Yan Z, et al. Gender-specific associations between atherogenic index of plasma and the presence and severity of acute coronary syndrome in very young adults: a hospital-based observational study. Lipids Health Dis. 2019;18(1):99. https://doi.org/10.1186/ s12944-019-1043-2.

5. Hu GL, Liu J, Liu J, Hao YC, Yang N, Zhou MG, et al. Association between smoking and the severity of coronary lesions among young and middleaged female patients with acute coronary syndrome. Zhonghua Xin Xue Guan Bing Za Zhi. 2020;48(5):378-85. https://doi.org/10.3760/cma.j.cn112 148-20191115-00702.

6. Li S, Pan G, Chen H, Niu X. Determination of serum homocysteine and hypersensitive $\mathrm{C}$-reactive protein and their correlation with premature coronary heart disease. Heart Surg Forum. 2019;22(3):E215-7. https://doi. org/10.1532/hsf.2223

7. Haj Mouhamed D, Ezzaher A, Neffati F, Douki W, Najjar MF. Effect of cigarette smoking on plasma homocysteine concentrations. Clin Chem Lab Med. 2011;49(3):479-83. https://doi.org/10.1515/CCLM.2011.062.

8. Chen S, Wu P, Zhou L, Shen Y, Li Y, Song H. Relationship between increase of serum homocysteine caused by smoking and oxidative damage in elderly patients with cardiovascular disease. Int J Clin Exp Med. 2015;8(3):4446-54.

9. Chinese Society of Cardiology of Chinese Medical Association, Editorial Board of Chinese Journal of Cardiology. Chinese guidelines for prevention of cardiovascular diseases. Zhonghua Xin Xue Guan Bing Za Zhi. 2011;39(1):3-22.

10. Alkerwi A, Baydarlioglu B, Sauvageot N, Stranges S, Lemmens P, Shivappa $\mathrm{N}$, et al. Smoking status is inversely associated with overall diet quality: findings from the ORISCAV-LUX study. Clin Nutr. 2017;36(5):1275-82. https://doi.org/10.1016/j.clnu.2016.08.013.

11. Mansia G, De Backer G, Dominiczak A, Cifkova R, Fagard R, Germano G, et al. 2007 ESH-ESC Guidelines for the management of arterial hypertension: the task force for the management of arterial hypertension of the European Society of Hypertension (ESH) and of the European Society of Cardiology (ESC). Blood Press. 2007;16:135-232. https://doi.org/10.1080/ 08037050701461084

12. Expert Committee on the Diagnosis and Classification of Diabetes Mellitus. Report of the expert committee on the diagnosis and classification of diabetes mellitus. Diabetes Care. 2003;26(1):S5-20. https://doi.org/10. 2337/diacare.26.2007.s5

13. Joint committee issued Chinese guideline for the management of dyslipidemia in adults. 2016 Chinese guideline for the management of dyslipidemia in adults. Zhonghua Xin Xue Guan Bing Za Zhi. 2016;44(10):833-53. https://doi.org/10.3760/cma.j.jissn.0253-3758.2016. 10.005 .

14. Guo LX. Interpretation of the Chinese expert consensus: Recommendations for diagnosis and treatment of asymptomatic hyperuricemia complicated with cardiovascular diseases. Chin J Pract Intern Med. 2014;2(2):93-6.

15. Rampidis GP, Benetos G, Benz DC, Giannopoulos AA, Buechel RR. A guide for Gensini Score calculation. Atherosclerosis. 2019;287:181-3. https://doi. org/10.1016/j.atherosclerosis.2019.05.012.

16. Maroszyńska-Dmoch EM, Wożakowska-Kapłon B. Clinical and angiographic characteristics of coronary artery disease in young adults: a single centre study. Kardiol Pol. 2016;74(4):314-21. https://doi.org/10.5603/KP. a2015.0178.
17. Christus T, Shukkur AM, Rashdan I, Koshy T, Alanbaei M, Zubaid M, et al. Coronary artery disease in patients aged 35 or less - A different beast? Heart Views. 2011;12(1):7-11. https://doi.org/10.4103/1995-705X.81550

18. Yano M, Miura S, Shiga Y, Miyase Y, Suematsu Y, Norimatsu K, et al. Association between smoking habits and severity of coronary stenosis as assessed by coronary computed tomography angiography. Heart Vessels. 2016;31(7):1061-8. https://doi.org/10.1007/s00380-015-0716-7.

19. Shenoy V, Mehendale V, Prabhu K, Shetty R, Rao P. Correlation of serum homocysteine levels with the severity of coronary artery disease. Indian J Clin Biochem. 2014;29:339-44. https://doi.org/10.1007/ s12291-013-0373-5.

20. Ding N, Sang Y, Chen J, Ballew SH, Kalbaugh CA, Salameh MJ, et al. Cigarette smoking, smoking cessation, and long-term risk of 3 major atherosclerotic diseases. J Am Coll Cardiol. 2019;74(4):498-507. https:// doi.org/10.1016/j.jacc.2019.05.049.

21. Chrysant SG, Chrysant GS. The current status of homocysteine as a risk factor for cardiovascular disease: a mini review. Expert Rev Cardiovasc Ther. 2018;16:559-65. https://doi.org/10.1080/14779072.2018.1497974.

22. van Meurs JB, Pare G, Schwartz SM, Hazra A, Tanaka T, Vermeulen SH. Common genetic loci influencing plasma homocysteine concentrations and their effect on risk of coronary artery disease. Am J Clin Nutr. 2013;98(3):668-76. https://doi.org/10.3945/ajcn.112.044545.

23. Sun J, Han W, Wu S, Jia S, Yan Z, Guo Y. Associations between hyperhomocysteinemia and the presence and severity of acute coronary syndrome in young adults $\leq 35$ years of age. BMC Cardiovasc Disord. 2021;21(1):47. https://doi.org/10.1186/s12872-021-01869-y.

24. Karadeniz M, Sarak T, Duran M, Alp C, Kandemir H, Etem Celik I, et al. Hyperhomocysteinemia predicts the severity of coronary artery disease as determined by the SYNTAX score in patients with acute coronary syndrome. Acta Cardiol Sin. 2018;34(6):458-63. https://doi.org/10.6515/ ACS.20181134(6).20180528B.

25. Hu R, Zhang XX, Wang WQ, Lau CP, Tse HF. Smoking, homocysteine and degree of arteriolar retinopathy. Atherosclerosis. 2005;183(1):95-100. https://doi.org/10.1016/j.atherosclerosis.2005.01.053.

26. O'Callaghan P, Meleady R, Fitzgerald T, Graham I; European COMAC group. Smoking and plasma homocysteine. Eur Heart J. 2002;23(20):1580-6. https://doi.org/10.1053/euhj.2002.3172.

27. Balint B, Jepchumba VK, Guéant JL, Guéant-Rodriguez RM. Mechanisms of homocysteine-induced damage to the endothelial, medial and adventitial layers of the arterial wall. Biochimie. 2020;173:100-6. https://doi.org/ 10.1016/j.biochi.2020.02.012.

28. d'Emmanuele di Villa Bianca R, Mitidieri E, Di Minno MN, Kirkby NS, Warner TD, Di Minno G, et al. Hydrogen sulphide pathway contributes to the enhanced human platelet aggregation in hyperhomocysteinemia. Proc Natl Acad Sci USA. 2013;110(39):15812-7. https://doi.org/10.1073/ pnas. 1309049110.

29. Yun J, Kim JY, Kim OY, Jang Y, Chae JS, Kwak JH, et al. Associations of plasma homocysteine level with brachial-ankle pulse wave velocity, LDL atherogenicity, and inflammation profile in healthy men. Nutr Metab Cardiovasc Dis. 2011;21(2):136-43. https://doi.org/10.1016/j.numecd. 2009.08.003.

30. Sinning C, Lillpopp L, Appelbaum S, Ojeda F, Zeller T, Schnabel R, et al. Angiographic score assessment improves cardiovascular risk prediction: the clinical value of SYNTAX and Gensini application. Clin Res Cardiol. 2013;102(7):495-503. https://doi.org/10.1007/s00392-013-0555-4.

\section{Publisher's Note}

Springer Nature remains neutral with regard to jurisdictional claims in published maps and institutional affiliations. 